\title{
Developing an International Registry for Uterus Transplantation (IRUTx):
}

\author{
Promises and Challenges
}

\section{NATASHA HAMMOND-BROWNING ${ }^{1} \&$ NICOLA JANE WILLIAMS $^{2},{ }^{*}$,}

ABSTRACT: Uterus Transplantation is an experimental vascular composite allograft designed to provide women with absolute uterine factor infertility with the opportunity to gestate and give birth to their future offspring. The number of UTx procedures performed worldwide now stand at $\geq 70$ and as the number of cases performed increases so too does the volume of the potential data that may be gathered to inform the development, practice, and regulation of UTx. Given the value of this data, and the challenges associated with keeping track of cases and outcomes where data remains unpublished and/or scattered, scientists and academics conducting research into UTx have increasingly called for the swift creation, implementation, and management of an international registry for Uterus Transplantation (IRUTx). This paper explores and provides practical guidance regarding the potential benefits the IRUTx may provide to stakeholders, as well as the legal and ethical challenges that its creation may pose in terms of dataset design, consent, privacy, researcher compliance, and governance.

\section{Introduction}

Uterus Transplantation (UTx) is an experimental vascular composite allograft (VCA) which is designed to provide women with absolute uterine factor infertility (AUFI) with the opportunity to gestate and give birth to their future offspring. Research into the procedure began using animal models in the 1960's (Eraslan et al, 1966), with the first attempt at performing the procedure in humans taking place in Saudi Arabia in 2000 (Fageeh et al, 2002). Since then, over 70 procedures have taken place globally in countries including Turkey, Sweden, China, the USA, the Czech Republic, Germany, Serbia, Brazil, India, France, and Lebanon and at least 20 births have now been reported (International Society of Uterus Transplantation, 2020) These numbers are increasing rapidly such that both

\footnotetext{
${ }^{1}$ School of Law and Politics, Cardiff University, Cardiff, UK

${ }^{2}$ Department of Politics, Philosophy and Religion, Lancaster University, Lancaster, UK.

*Correspondence: Nicola Jane Williams, Department of Politics, Philosophy and Religion, County South Lancaster University, Lancaster, UK, LA1 4YW. Email n.williams2@,lancaster.ac.uk; Natasha Hammond-Browning, School of Law and Politics, Cardiff University, Law Building, Museum Avenue, Cardiff, CF10 3AX. Email HammondBrowningN@,cardiff.ac.uk
} 
procedures and births doubled between 2016 and 2018, from 25-52, and 5-10 respectively (Jones et al, 2019: 1311), and additional trials are currently being planned in Australia, Belgium, Japan, Sweden, and the UK.

29 As the number of cases performed increases so too does the volume of potential data that may be gathered in both the short and long-term to inform the development, practice, and regulation of UTx. Given the value of such data, along with the challenges of keeping track of cases and outcomes, especially where data remains unpublished and/or scattered, scientists and academics conducting research into UTx have increasingly called for the swift creation, implementation, and management of "an international registry of uterus transplantation cases with follow-up of patients, children and donors." (International Society of Uterus Transplantation, 2017) The establishment of this registry has now been announced, but it is still in the development stages, with little information currently available regarding its proposed aims, scope. ${ }^{3}$ In what follows we - through analysis of the ethical, scientific and legal literature surrounding medical registries and work undertaken by the International Society for Uterus Transplantation (ISUTx) - explore: the potential benefits for both physicians, patients, and wider stakeholders that may be provided by the creation of an International Registry for Uterus Transplantation (IRUTx); how to determine the kinds of information that should be collected and collated; and a number of ethical and legal challenges that the development of an international registry for UTx may pose in terms of consent, privacy, researcher compliance, and governance.

\section{2. Designing the International Registry for Uterus Transplantation: Aims and Scope}

In late 2017, during the ' 1 st Congress of International Society for Uterus Transplantation (ISUTx)', the then ISUTx President and leader of the Swedish uterus transplantation team, Professor Mats Brännström, together with Dr. César Díaz García, presented the Society's proposal to design and manage an international registry of uterus transplantation cases. (International Society of Uterus Transplantation, 2017) They set forth the first detailed plans available for the design and development of what they described as a "Patient-based, user-friendly, centralized, secure, anonymous, exhaustive and specific" registry of UTx cases. (Brännström et al, 2017) Their stated aims for the registry were to provide "a strong body of knowledge" with "validated data on: efficacy, safety [and] other outcomes" (Brännström, et al, 2017) for donors, recipients and children born through UTx. An update on the Society's progress and a first look at an extensive draft version of the registry was provided a year later at the ' 1 st State of the Art Meeting of ISUTx'

\footnotetext{
TDr's Hammond-Browning and Williams consider that they should be regarded as joint first authors.

${ }^{3}$ See section 2 for further details
} 
54 (International Society of Uterus Transplantation, 2018) The Society's intentions for the registry were then confirmed

55 in a 2019 paper which stated:

56

57

A global UTx registry, containing data in relation to donor, recipient, surgery, immunosuppression, and pregnancy, has been created by ISUTx. Future annual reports and registry-based research studies will provide results on activity and scientific results within this emerging field of infertility treatment, with major elements of both reproductive surgery and assisted reproduction. (Brännström et al, 2019:9)

The launch of the registry was then confirmed in a newsletter distributed to the members of ISUTx from the Society in January 2020. However, as has been confirmed by private communication with the President and past President of the Society, the registry is, at the time of writing this paper (April 2020) still under construction and there is very little publicly available information regarding its planned aims and scope. We argue that it is vital for ISUTx to establish the purpose of the registry from the outset as it is only once the purpose and goals of the registry are determined that the data required to achieve these goals can be identified (PARENT Cross border Patient Registries iNiTiative. 2015:6.3)From the little information that is available it can be noted that the proposed scope of the registry seems rather broad with plans to utilize the information of which it is comprised for research, educational, and oversight purposes by or for the benefit of) a number of different possible stakeholder groups, such as donors, recipients, future beneficiaries of UTx research, women with AUFI, children created through UTx, physicians/teams conducting/planning to conduct UTx trials, psychologists, regulators, funders, journalists, and academics. A broad scope is not necessarily disadvantageous. For, clearly defined and well managed registries serving multiple purposes are able to provide greater benefits for stakeholders than those with a more limited remit. In the context of the IRUTx these benefits are likely to include (but may not be limited to):

- Increases in data reliability through reduction of challenges for patient safety, and research and development, associated with reporting biases such as publication and time lag bias; and the use of different measurement tools for the same/similar outcomes through standardization of data reported;

- The production (and encouragement) of more and better-informed comparative research due to access to higher quality, centralized, and standardized data on existing cases as well as published literature;

- Greater awareness of AUFI and UTx among members of the public and journalists through information disseminated on the registry website, and newsletters. 
- Greater levels of understanding of AUFI and UTx among stakeholders lacking access to the academic literature or unpublished data such as journalists and members of the public resulting from the availability of up to date, verified, and centrally located information (in the form of annual reports of key outcomes).

- Higher quality patient consent due to ability of individual research teams to more accurately inform patients about current state of knowledge of risks and benefits of UTx;

- Better patient safeguarding through faster identification of technique variations/patient groups/teams/individual physicians etc. demonstrating poor patient outcomes; and/or

- Reductions in duration of the surgical learning curve for teams preparing to trial the procedure and subsequent reductions in patient risks associated with this. ${ }^{4}$

To achieve such a wide range of benefits, however, the data collected and collated in the IRUTx must be sufficiently complete, accurate, and relevant to the interests of the stakeholders it serves, and wholly compliant with national and international guidelines regarding data protection and privacy. It is therefore imperative, in order to ensure that the time, effort, resources, and information funnelled into establishing and operating the registry are not wasted, that the IRUTx is carefully designed to maximise the chances that it will embody these four characteristics. Completeness and accuracy will, of course, primarily be achieved through the management, funding, and population of the registry once launched. Steps, however, can be taken at the design stage of the registry to increase their likelihood such as through a clear management structure; the creation of viable funding strategies; and ensuring easy access, an intuitive end userinterface for those depositing data in the registry.

Ensuring relevance for stakeholders and/or deciding which potential stakeholders the registry should cater for, however, requires significant effort at the design stage as it can be noted that once the registry is up and running it may prove difficult, costly, and time consuming to add and complete datasets later stage. The initial process of selecting and building data sets may determine the final success of the IRUTx and is one of the most important and challenging tasks of a new registry (Zaletel et al, 2015:s. 6.3). For, in determining the datasets to include in the IRUTx those designing it must seriously consider a number of practical and ethical considerations regarding: the diverse priorities and interests of different stakeholder groups, and their views regarding the relative importance of including different datasets; the weightiness of the interests and preferences of different stakeholders regarding the inclusion/exclusion

\footnotetext{
${ }^{4}$ These suggested benefits are partially based on those suggested by Brännström and García in their 2017 presentation at the $1^{\text {st }}$ Congress of the ISUTx (cited previously).
} 
112 of different datasets; and, the level of priority (if any) to accord to the interests of those who contribute to the registry

113 or are most burdened by its creation such as patients (who provide their data), UTx teams (who record their cases),

114 and those who fund the registry.

115

116 Those designing the IRUTx will therefore be forced to acknowledge and explore how different prospective registry

117 designs could serve to advance or set back the interests of different stakeholder groups, and engage the key ethical

118 principles often raised by research involving human volunteers: respect for persons, beneficence/non-maleficence,

119 and justice (National Commission for the Protection of Human Subjects of Biomedical and Behavioural Research,

120 1978). A key question in such discussions will likely be how to balance the privacy and welfare interests of those whose

121 personal information is included in the registry (donors, recipients and children) against the welfare interests of future

122 beneficiaries of UTx research, and the interests of researchers and funders. For, while a concern for the interests of

123 the former group generally motivates consideration of necessity and the limitation of datasets included in the registry

124 a concern to maximise benefits for the latter tends to push in the opposite direction. How to balance such considerations and settle on a registry design that is acceptable to all stakeholders has been the subject of some

126 discussion in the literature surrounding clinical trials where initiatives like SONG (standardized outcomes in 127 Nephrology), OMERACT (outcome measures in Rheumatology)and COMET (Core Outcome Measures in 128 Effectiveness Trials) have proposed a number of methods for establishing core datasets for clinical trials (SONG 129 Initiative, 2017; Boers er al, 2014; Williamson et al, 2017) These may prove useful in the design of the IRUTx and 130 have included: systematic literature reviews; the use of focus groups; stakeholder interviews; Delphi Surveys; and

131 Consensus Workshops (SONG Initiative, 2017:7)

\section{3. Governance and Oversight}

135 In order to foster professional and public confidence in the IRUTx - which should, in turn, encourage participation

136 from patients, and physicians conducting research into UTx - significant care should be taken to ensure transparent

137 and egalitarian governance and oversight of the registry and its activities. These should be codified, reflect the nature

138 and extent of the registry's operations from the outset, be suitably representative of different key stakeholder groups,

139 and ensure effective registry operation and longevity. The early establishment of a governance plan will assist in the

140 design and maintenance of the IRUTx (Zaletel et al, 2015:s6.1). Given the small size of the registry it is likely that the

141 majority of IRUTx governance and oversight activities - such as those regarding day-to-day finances and

142 administration, funding, liaison with stakeholders, registry maintenance, quality assurance and data-cleaning 
143 procedures, registry reports, and data access, use and publication (Gliklich et al, 2014:2.5) will be undertaken and

144 determined by the registry's executive/steering committee.

146 The executive/steering committee should be comprised of members of various different stakeholder groups to ensure

147 that the perspectives of all with the greatest stake in the registry's interests and perspectives are taken into account.

148 Given the many and complex jobs of the executive/steering committee it is also imperative that it consult with relevant

149 experts from finance, ethics, law and biostatistics when required to ensure best practice and compliance with national

150 and international legislation. In order to increase accountability to the wider stakeholders the IRUTx serves and to

151 allow the committee's members to benefit from the advice of a wider community of experts and stakeholders it is also

152 advisable that an Independent Advisory Board (or equivalent) is formed (Gliklich et al, 2014:4.2). With all the

153 operations of the IRUTx, transparency, openness and visibility is key; from the members and role of the

154 executive/steering committee, the policies, and consent processes, through to the purposes of data collection, data

155 protection procedures, and research protocols (amongst others). The creation of a website which includes public

156 information regarding processes for appointing members, clear objectives, a clear request process for those who seek

157 to access IRUTx data for their own research work, and details about the funding of the IRUTx will assist in making

158 the operations of the registry transparent, and correspondingly increase 'confidence in the scientific integrity and

159 validity of registry processes, and therefore in the conclusions reached as a result of registry activities.' (Gliklich et al,

160 2014:4.1).

161

162 As noted previously, it is also imperative to maximise the benefits that the IRUTx can provide to potential stakeholders

163 while correspondingly minimizing harms. In service of this aim public information about registry operations could

164 significantly increase "the scientific utility of registry data by promoting inquiries from scientists with interests to

165 which registry data may apply." (Gliklich et al, 2014:4.1) Fair and equitable policies must be formulated and clearly

166 articulated on the registry's website and code of practice regarding both the publication of research arising from

167 registry activities and researcher access to raw registry data. For, while the prospect of data sharing understandably

168 raises concerns regarding data ownership, consent, privacy, and data protection (explored below), the scientific utility

169 of the IRUTx will be increased if submission of requests to access registry data from a broad range of disciplines and

170 researchers is actively encouraged. A significant problem noted by those who manage medical registries in the past

171 has been that of the underutilization of registry data and the number of research questions which could, but are not,

172 explored due to limits in terms of time, expertise and personnel. (Dane et al, 2006) Thus, while those who maintain

173 or fund the IRUTx may have proprietary interests in first publication/monetization of the registry and may seek to 
174

175

176

177

178

179

180

181

182

183

184

185

186

187

188

189

190

191

192

193

194

195

196

197

198

199

200

201

202

203

embargo data for a certain period of time, such interests should be balanced against the potential benefits that may be achieved by widening access to registry data as soon as possible.

Transparent governance and oversight, fair and equitable policies regarding access to data, and clear articulation of the benefits that this registry could produce should also increase professional confidence in the IRUTx. In turn, this should encourage physicians to engage with the IRUTx through populating the registry with the necessary data. Compliance is vital in order to create and maintain a sufficiently complete and accurate registry and avoid the introduction of resulting bias. However, as the IRUTx will not be established by primary national or international legislation there is no legal basis though which compliance can be compelled. Ethical question marks also arise if actively coercive or manipulative activities are undertaken to increase compliance from physicians such as through denial of requests to access data for research purposes or expulsion from the ISUTx. Such activities could, after-all, have serious and negative effects on patient welfare, safety, and consent to inclusion in the registry. Given the association of the IRUTx with the ISUTx, however, the Society's soft power could be harnessed to encourage compliance from society members through reminders in newsletters, promotion at the annual ISUTx conference, etc.

Careful consideration of how to ensure stable, appropriate, and sustainable funding sources and strategies is also crucial at this initial planning stage. With a desire for long-term follow-up studies of children born from a donated uterus, as well as recipients and donors, the continuing financial stability of the IRUTx along with the maintenance of the datasets that it will hold is of significant concern. The costs associated with populating, maintaining and managing the registry over the long term should not be underestimated and there is a pressing need to maximise funding and identify sustainable funding sources able to provide long-term support to ensure the IRUTx is able to meet its goals. Finally, it is imperative that IRUTx funders and the conditions attached to their support are disclosed and visible to all stakeholders and that sources of funding are carefully vetted by the registry's executive/steering committee. The presence of ethical concerns regarding funder activities or worries about conflicts of interest and/or inappropriate levels of influence over registry activities may, after-all, impact levels of confidence in outputs produced from registry data and ultimately threaten the long-term viability of the IRUTx. This is especially pressing where funding is obtained from non-governmental sources such as patient groups, private companies and foundations who may have a vested interest in UTx. 
In order to ensure public confidence in the IRUTx, it is important that the IRUTx is a transparent body that upholds ethical principles. Openness as to what information is deposited with it, who can access that information, and how it will be used must be a central principle of the IRUTx.

209 The IRUTx may have several roles to fulfil, and these, as noted previously, are likely to include reporting and research.

210 However, the IRUTx could also expand to encompass broader informational, educational and advisory roles which,

211 as noted previously, may significantly increase the prospective benefits provided by the registry. An informational role

212 would require the IRUTx to be public as well as private facing, providing annual updates to the public that may include 213 information such as the number of uterus transplants performed worldwide, pregnancies, and births recorded. The

214 IRUTx could also provide up-to-date public facing information about the techniques utilised in UTx, the risks for 215 donors and recipients, and the alternatives to UTx. This would be similar to the information provided by the United

216 Kingdom's Human Fertilisation and Embryology Authority in the context of fertility treatment more generally 217 (Human Fertilisation and Embryology Authority, 2017). The IRUTx could also have an educational role and registry 218 outcomes may be used to update and train physicians and other medical professionals conducting and preparing to 219 conduct UTx trials, and other stakeholders on specific developments. This may be through annual reports, creation of educational materials using registry data, and information dissemination on an ad hoc basis to update practice (such as where registry data disrupts clinical equipoise between different surgical techniques being trialled). Finally, the IRUTx may also perform an advisory role. As an international registry it could provide updates to governments, policy makers, regulators, and governing bodies in order to assist with the regulation of uterus transplantation. As such, the data that the IRUTx will need to be provided with by researchers and practitioners could be extensive. Furthermore, in order to fulfil these roles, the IRUTx will be dependent upon accurate and timely reporting, which in turn is reliant upon participant's initial and ongoing consent to the deposit of data in the IRUTx.

228 As noted previously, discussions with stakeholders will help to inform decisions regarding key datasets and to reduce 229 the collection of unnecessary data. These datasets will be of considerable value; to assist the IRUTx in its public facing, informational and policy role, and importantly to help to inform and educate physicians working in UTx. The role of consent is central to establishing good working practices of the IRUTx, and to enable it to fulfil its aims. It may, therefore, be argued that there is a need for a single reporting process which would encompass both a notification procedure, and a more detailed dataset. The notification procedure may be compared to any other medical treatment that mandates compulsory reporting, such as termination of pregnancies in England and Wales. For example, physicians would report that a transplant has taken place, the type of donor, and the outcome of that transplant. It 
would be strongly encouraged that all physicians working in UTx comply with this notification procedure, thereby assisting in the accurate compilation of procedures and outcomes. A more detailed dataset would assist the IRUTx in its educational role, as well as providing information for more detailed statistical reporting. However, not all participants in a UTx trial may be willing for detailed personal data to be transferred to the IRUTx and an autonomous decision not to consent to the inclusion of their datasets should be respected. The compilation of large datasets is, after-all, not without its risks, and some participants may be understandably reluctant to transfer personal data to a central Registry.

The major risk of depositing detailed data within a central Registry is that the donors and recipients may be easily identifiable, even when data is anonymised. This concern is especially compelling in the context of uterus transplantation given the relatively small pool of potential recipients and media interest in the procedure. For example, previous recipients have been identified in the media either in press releases from centres that have performed UTx, or in interviews post-birth (BBC News, 2020). Information disclosed could then be matched with the anonymised data in the Registry. This risk of identification will reduce with time and corresponding increases in recipient numbers. However, the risk of privacy breaches must not be underestimated, and UTx recipients should be informed of such risks prior to consenting to the deposit of their data within the registry to ensure that they are in receipt of sufficient information to make an informed choice regarding participation.

254 The IRUTx will therefore need to establish procedures to ensure that appropriate consent of participants has been obtained prior to their data being deposited with the IRUTx. It is important that physicians worldwide review the consent forms signed by recipients and donors prior to the establishment of the IRUTx, to ensure that the transferral of data to a central international registry is within the scope of the consent given. It is also vital that participants in UTx have the opportunity to withdraw consent to data sharing. It must not be presumed that broad consent to participate in research will also cover the transference of data to an international registry. An interactive consent model that allows participants to vary their consent preferences would allow participants greater control over their personal data (Holm et al, 2019). Moving forward, it is recommended that the IRUTx establish a standardised consent form for participants with regard to the sharing of their data with the IRUTx, and the purposes for which that data is being stored, as well as establishing an interactive consent model. Participant's consent to involvement in long-term follow up studies must also be considered; an interactive consent model could encourage participation in long-term studies as participants may feel in control of their data. Researchers desire access to data held by the IRUTx in order to 
on participants of UTx are also to be encouraged. For only longitudinal studies will establish the long-term safety of the procedure and the health and wellbeing of recipients, donors, and children born. ${ }^{5}$

As noted previously, unlike bodies established through primary legislation, such as the United Kingdom's Human

271 Fertilisation and Embryology Authority (Human Fertilisation and Embryology Act 1990), the IRUTx will only have

272 persuasive powers to encourage physicians to deposit the required data into the Registry on a regular basis. The IRUTx

273 will play a vital role in stressing the importance to researchers of establishing core datasets, and that submissions

274 should include core data in order to assist with this process. If data collection and deposit are seen to be arduous and time-consuming tasks, they are less likely to be completed, so ease of access is significant. The applicable governing

276 law must be considered and communicated to physicians and participants in UTx; this is important for both the

277 IRUTx and those who deposit data within it. Privacy concerns have increased in recent years, and it is therefore

278 essential that the IRUTx sufficiently respects and upholds the privacy of those whose data is submitted to and included

279 in the registry, as well as ensuring that anyone who submits and/or is able to access the data complies with the relevant

280 law. Sanctions for those who breach the relevant law must also be clear, and enforceable. Similar bodies in other contexts, such as the UK's Stem Cell Bank, have been criticised on these grounds (Hammond-Browning et al, 2013).

The European Union's (EU) General Data Protection Regulation (Regulation (EU) No 2016/679) is the strictest set of data protection regulations worldwide and could provide a robust benchmark for the Registry. A key aim of the GDPR is to make data protection 'by design and by default' throughout its lifecycle (Information Comissioner's Office, 2019:197-205). It provides legal safeguards for personal data within the EEA and the UK ${ }^{6}$ regardless of where the processing of the data takes place. The GDPR will already apply to public and private institutions performing UTx within the EEA and UK. Significantly, GDPR restricts the transfer of personal data outside the EEA (even for processing) unless the country in question can demonstrate that the rights of individuals are adequately protected (General Data Protection Regulation, Art. 45; Information Commissioner's Office, 2019: 260-277). Practical guidance on compliance with the GDPR, where personal data is transferred and disclosed between organisations, can be found in the Code of Practice produced by the UK's designated enforcement body, the Information Commissioner's Office.

\footnotetext{
${ }^{5}$ For example, long-term studies by S Golombok and the team at the Centre for Family Research, Cambridge University, have helped significantly to inform the debate around surrogacy and new families.

${ }^{6}$ The EEA consists of the 27 EU countries and Iceland, Liechtenstein and Norway. EU law remains applicable in its entirety in the UK until the end of the transition period (which is currently 31.12 .20 with the possibility of being extended for one or two years). After exit day, the GDPR will become part of UK law (the UK GDPR as part of 'EUderived law) in accordance with the EU (Withdrawal) Act 2018. However, it may be subject to changes by Ministers or Parliament. Negotiations continue on UK-EU transfers and how the UK will provide 'adequate protection' to safeguard data protection rights originating in the EU
} 
It states:

The most important thing is to ensure that the organisations involved in data sharing work together to ensure that the individuals concerned know who has, or will have, their data and what it is being used for, or will be

\section{1}

302

This reinforces the necessity of appropriate consent from participants in UTx before data is deposited with the IRUTx, and the need for consent before data is shared beyond the IRUTx. As such, accessing data held by the Registry by parties outside of the EEA requires consideration and planning by the founders of the IRUTx. Enforcement is decentralised to national level: Member States must establish a supervisory authority that has the competence to exercise the powers conferred within the GDPR, including complaints and investigations on the application of the GDPR (Regulation (EU) No 2016/679: Art. 51 \& 55). The sanctions that can be imposed for infringements can be onerous ranging up to $€ 20$ million, or 4\% revenue whichever is higher (Regulation (EU) No 2016/679: Art. 83). However, sanctions may be more difficult to impose where a breach occurs in a non-EEA (or third country)..

310 Moreover, individuals whose data protection rights are infringed will be able to seek a remedy before the courts 311 (Regulation (EU) No 2016/679: Art. 79).

\section{Conclusion}

\section{4}

315 The value of a registry for UTx is not without doubt. What is apparent is the need for careful design of its aims, 316 governance, funding, and policies, reasonable strategies to encourage physician compliance and the provision of 317 relevant training in order to ensure compliance with data privacy laws, and maximise stakeholder benefits. The 318 initiative taken by the International Society for Uterus Transplantation to set up an international registry is welcomed 319 and a wide range of benefits may be reaped from the near-future establishment of the IRUTx. A note of caution must, however, be aired; that the autonomy of participants must be respected, and time is taken to design datasets to ensure that they are complete, accurate, and relevant to stakeholders, as well as legally compliant. We have argued that there is a need to establish the goals and purposes of the IRUTx from the outset in order to determine the required datasets 
to obtain initial and ongoing consent of participants, with the development of an interactive consent model; and compliance by all parties with GDPR.

- Identify key stakeholders and ensure sensitivity to and awareness of their differing priorities and interests, including conflicts of interests between members of different groups. minimises potential harms to and maximises benefits. guidelines regarding data protection and privacy experts

- Ensure and maintain transparency in disclosure of funding sources, policies, purposes of research, members, research protocols Create website that includes public facing information and contact details for researchers

- Design and establish consent and data protection policies and procedures for data deposit to IRUTx, to include:

$>$ Review of consent provided to data collection and transfer prior to the establishment of IRUTx

$>$ Establish interactive consent model - allow for withdrawal of consent and encourage participation in long-term studies. Policies to include information on applicable governing law, compliance, enforcement and sanctions. GDPR governs transfer of data within and out with the EU. 


\section{Funding}

Dr. Williams' research is funded by a Leverhulme Trust Early Career Fellowship in the Arts and Humanities (Grant no: ECF-2018-113 Title: Quality of Life Transplantation - Philosophical and Policy Questions.

\section{Acknowledgments}

Dr. Hammond-Browning would like to thank members of her Coffee, Research and Pastries group for their invaluable comments and feedback on earlier drafts of this paper: Louise Austin, Dr Roxanna Dehaghani, Dr Sara Drake, Dr Anna Heenan, and Caer Smyth. Dr Williams would like to thank the Leverhulme Trust and members of the Reproductive Donation Group at Lancaster University (Professor Stephen Wilkinson and Laura O'Donovan in particular) who have provided invaluable support for her research.

\section{Bibliography}

BBC News. 'Miracle Baby Born After Womb Transplant'. 3/01/2020. Available at: https://www.bbc.co.uk/news/av/world-us-canada-51100109/miracle-baby-born-after-womb-transplant $[$ [last accessed 11/05/2020]

Boers M, Kirwan JR, Wells G, Beaton D, Gossec L, d'Agostino MA, Conaghan PG, Bingham CO 3rd, Brooks P, Landewé R, March L, Simon LS, Singh JA, Strand V, Tugwell P. Developing core outcome measuremdng sets for clinical trials: OMERACT filter 2.0. J Clin Epidemiol 2014 67(7): 745-53.

Brännström M, Enskog A, Kvarnström N, Ayoubi JM, Dahm-Kähler P. Global Results of Human Uterine Transplantation and Strategies for Pre-Transplantation Screening of Donors. Fertil Steril 2019 112(1): 3-10 .

Brännström M, Diaz Garcia C. Why a UTx Registry and How Will Results Be Used'. Presentation, International Society For Uterus Transplantation. 1st Congress of ISUTx: Scientific Program. 2017 Available at: http://www.isutx.org/wp-content/uploads/2017/11/Scientific-program-ISUTx-2.pdf [last accessed 11/05/2019].

Dane FC, Parish DC. Ethical Issues in Registry Research: In-Hospital Resuscitation as a case study. J Empir Res Hum Res Eth 2006 1(4):69-76. 
Eraslan S, Hamernik RJ, and Hardy JD. Replantation of uterus and ovaries in dogs, with successful pregnancy. Arch

Surg 1966. 92(1):9-12.

417 Fageeh W, Raffa H, Jabbad H, Marzouki A. Transplantation of the human uterus. Int J Gynaecol Obstet 200276 (3):

418 245-51.

Gliklich RE, Dreyer NA, Leavy MB (eds). Registries for Evaluating Patient Outcomes: A User's Guide. 2014. $3^{\text {rd }}$ Ed, Rockville (MD): Agency for Healthcare Research and Quality (US); 2014 Apr. Report No.: 13(14)-EHC111.

Hammond-Browning N, Stephens N. Moving Human Embryonic Stem Cells Internationally: Near-future Challenges for the UK Stem Cell Bank and American Collaborators in N Priaulx \& A Wrigley (eds.) Ethics Law and Society. 2013. London: Routledge: 299-313.

Holm S, Ploug T. Genome Studies Reveal Flaws in Broad Consent.' Science 2019366 (6472):1460-61.

Human Fertilisation and Embryology Authority. Our Strategy 2017-2020. 2017 HFEA: London. Available at: https://ifqlive.blob.core.windows.net/umbraco-website/1346/hfea strategy-2017 aw.pdf [last accessed $11 / 05 / 2020]$.

Information Commissioner's Office. Guide to the General Data Protection Regulation (GDPR). 2019 ICO: London. Available at: https://ico.org.uk/media/for-organisations/guide-to-data-protection/guide-to-the-general-dataprotection-regulation-gdpr-1-0.pdf [last accessed 11/05/2020].

Information Commissioner's Office. Data sharing code of practice. 2011 ICO: London. Available at: https://ico.org.uk/media/for-organisations/documents/1068/data sharing code of practice.pdf [last accessed $11 / 05 / 2020]$.

International Society of Uterus Transplantation. Newsletter: Private Communication to ISUTx members. 2020

International Society of Uterus Transplantation. '1st State Of The Art Meeting of ISUTx: Programme' 2018. Available at http://www.uterustransplantation.org/schedule [last accessed 11/05/2020].

International Society of Uterus Transplantation. ISUTx Bylaws. 2017. Available at: http://www.isutx.org/wpcontent/uploads/2017/11/ISUTx-Bylaws-2017.pdf [last accessed 11/05/2020].

International Society of Uterus Transplantation. ‘1st Congress of ISUTx: Scientific Program' 2017 Available at: http://www.isutx.org/wp-content/uploads/2017/11/Scientific-program-ISUTx-2.pdf [last accessed 11/05/2019].

Jones BP, Saso S, Bracewell-Milnes T, Thum MY, Nicopoullos J, Diaz-Garcia C, Friend P, Ghaem-Maghami S, Testa G, Johannesson L, Quiroga I, Yazbek J, Smith JR. Human Uterine Transplantation: A review of outcomes from the first 45 cases. BJOG 2019 126(11):1310-1319.

National Commission for the Protection of Human Subjects of Biomedical and Behavioural Research, Department of Health, Education and Welfare. The Belmont Report. 1978 Washington: United States Government Printing Office. 1978.

Regulation (EU) 2016/679 of the European Parliament and of the Council of 27 April 2016 on the protection of natural persons with regard to the processing of personal data and on the free movement of such data, and repealing Directive 95/46/EC (General Data Protection Regulation) (Text with EEA relevance) [2016] OJ L119/1.

SONG Initiative. The SONG Handbook. Version 1.0 June 2017, Sydney, Australia Available at www.songinitiative.org/reports-and-publications/ [last accessed 11/05/2020]

Williamson PR, Altman DG, Bagley H, Barnes KL, Blazeby JM, Brookes ST, Clarke M, Gargon E, Gorst S, Harman N, Kirkham JJ, McNair A, Prinsen CAC, Schmitt J, Terwee CB, Young B. The Comet Handbook version 1.0. Trials 2017 18(s3): 1-50.

470 Zaletel M, Kralj M (eds). Methodological guidelines and recommendations for efficient and rational governance of 471 patient registries. Ljubljana : National Institute of Public Health, 2015. 
